Revue internationale P.M.E.

Économie et gestion de la petite et moyenne entreprise

\title{
L'impact de la structure de la famille sur le développement des petites entreprises familiales en hypogroupe
}

\section{Imen Mzid Ben Amar et Lassaâd Mezghani}

Volume 23, numéro 1, 2010

URI : https://id.erudit.org/iderudit/045747ar

DOI : https://doi.org/10.7202/045747ar

Aller au sommaire du numéro

Éditeur(s)

Presses de l'Université du Québec

ISSN

0776-5436 (imprimé)

1918-9699 (numérique)

Découvrir la revue

Citer cet article

Ben Amar, I. M. \& Mezghani, L. (2010). L'impact de la structure de la famille sur le développement des petites entreprises familiales en hypogroupe. Revue internationale P.M.E., 23(1), 125-154. https://doi.org/10.7202/045747ar

\section{Résumé de l'article}

La structure de la famille est le système d'interactions au sein de la famille qui influence le comportement de chaque membre et sa perception de la réalité (Todd, 1983). La diversité des structures peut avoir une influence sur le développement stratégique des entreprises familiales. En fait, le développement des entreprises familiales, notamment de petite taille, introduit l'idée de l'organisation stratégique en hypogroupe susceptible de permettre la mise en oeuvre des stratégies de croissance par création de nouvelles unités. Ainsi, notre ambition, à travers cette recherche, est d'étudier l'impact de la structure de la famille sur le développement de l'entreprise familiale en hypogroupe.

L'information est collectée par le biais d'entretiens semi-directifs auprès des dirigeants propriétaires de cinq cas d'hypogroupes. Les données ont fait l'objet d'une analyse de contenu thématique. Nous avons pu conclure que l'organisation stratégique en hypogroupe est adoptée par les dirigeants qui souhaitent accorder à chacun des successeurs les mêmes opportunités pour réaliser les objectifs personnels de ces derniers. L'égalité entre les successeurs permet d'éviter les conflits internes qui peuvent peser sur la pérennité de l'entreprise. Le choix de l'activité de l'unité nouvellement créée dépend du profil du successeur. Étant donné que les successeurs ont des profils différents, le développement en hypogroupe se réalise à travers la diversification.
Ce document est protégé par la loi sur le droit d'auteur. L'utilisation des services d'Érudit (y compris la reproduction) est assujettie à sa politique d'utilisation que vous pouvez consulter en ligne.

https://apropos.erudit.org/fr/usagers/politique-dutilisation/ 


\title{
L'impact de la structure de la famille sur le développement des petites entreprises familiales en hypogroupe
}

\author{
Imen MZID BEN AMAR \\ Institut supérieur d'administration des entreprises \\ Université de Gafsa, Tunisie \\ Lassaâd MEZGHANI \\ Faculté des sciences économiques et de gestion \\ Université de Sfax, Tunisie
}

\section{MOTS CLÉS}

\section{Entreprise familiale - Structure de la famille - Égalité Hypogroupe - Diversification}

\begin{abstract}
LES AUTEURS
IMEN MZID BDN AMAR est assistante à l'Institut supérieur d'administration des entreprises de Gafsa en Tunisie. Outre de détenir un doctorat en sciences de gestion de l'Université de Toulon en France, elle est membre de l'unité de recherche en gestion des entreprises (URGE). Elle travaille sur les stratégies de développement des entreprises familiales et a rédigé certains articles sur ce thème. Elle enseigne la stratégie et la gestion des ressources humaines.

LASSAÂD MEZGHANI est professeur de management stratégique à la Faculté des sciences économiques et de gestion de l'Université de Sfax. II est titulaire d'un doctorat en management stratégique de l'École centrale Paris, d'un master en science et d'un baccalauréat en génie industriel de l'Université du Wisconsin à Madison, États-Unis. II enseigne la stratégie, le management de la technologie, les systèmes et technologies de l'information (STI) et la gestion de projet de STI. II dirige, au sein de l'URGE, des thèses de doctorat et des travaux de recherche portant sur les stratégies concurrentielles, les stratégies de développement des entreprises familiales, l'adoption des STI et, plus récemment, le management des milieux innovants (pépinières et technopôles). Il est membre du conseil de l'Université de Sfax et du conseil d'administration de l'Association internationale de management stratégique (AIMS).
\end{abstract}




\title{
RÉSUMÉ
}

La structure de la famille est le système d'interactions au sein de la famille qui influence le comportement de chaque membre et sa perception de la réalité (Todd, 1983). La diversité des structures peut avoir une influence sur le développement stratégique des entreprises familiales. En fait, le développement des entreprises familiales, notamment de petite taille, introduit l'idée de l'organisation stratégique en hypogroupe susceptible de permettre la mise en œuvre des stratégies de croissance par création de nouvelles unités. Ainsi, notre ambition, à travers cette recherche, est d'étudier l'impact de la structure de la famille sur le développement de l'entreprise familiale en hypogroupe.

L'information est collectée par le biais d'entretiens semi-directifs auprès des dirigeants propriétaires de cinq cas d'hypogroupes. Les données ont fait l'objet d'une analyse de contenu thématique. Nous avons pu conclure que l'organisation stratégique en hypogroupe est adoptée par les dirigeants qui souhaitent accorder à chacun des successeurs les mêmes opportunités pour réaliser les objectifs personnels de ces derniers. L'égalité entre les successeurs permet d'éviter les conflits internes qui peuvent peser sur la pérennité de l'entreprise. Le choix de l'activité de l'unité nouvellement créée dépend du profil du successeur. Étant donné que les successeurs ont des profils différents, le développement en hypogroupe se réalise à travers la diversification.

\begin{abstract}
The family's structure is the system of interactions within the family that influences the behavior of each member and his/her perception of reality (Todd, 1983). The diversity of structures can have an influence on the strategic development of family firms. In fact, the development of family firms, in particular of small size, suggests the idea of the strategic organization of these firms into a hypogroup that allows the implementation of growth strategies by creation of new units. Thus, our ambition, through this research, is to study the influence of the family's structure on the development of the family firm into a hypogroup.

Information is collected through semi-oriented interviews conducted with owner-managers of five cases of hypogroups. The data were subject to thematic content analysis (TCA). It was possible to conclude that the strategic organization into hypogroup was adopted by managers that wanted to grant each of the successors the same opportunities to achieve his/her personal goals. The equity between successors permits to avoid internal conflicts that can impact the family firm perenniality. The choice of the activity of the newly created unit depends on the profile of the successor. Given that successors have different profiles, the development into hypogroup is conducted through a diversification strategy.
\end{abstract}

\section{RESUMEN}

La estructura de la familia es el sistema de interacciones en el seno la familia que influye sobre el comportamiento de cada miembro y su percepción de la realidad (Todd, 1983). La diversidad de las estructuras puede tener una influencia sobre el 
desarrollo estratégico de las empresas familiares. De hecho, el desarrollo de las empresas familiares, en particular las de pequeño tamaño, introduce la idea de la organización estratégica en hipo-grupo susceptible de permitir la aplicación de las estrategias de crecimiento por creación de nuevas unidades. Así pues, nuestra ambición, a través de esta investigación, es estudiar el impacto de la estructura de la familia en el desarrollo de la empresa familiar en hipo-grupo.

La información se recoge por medio de conversaciones semidiretivas ante los dirigentes propietarios de cinco casos de hipo-grupos. Los datos fueron objeto de un análisis de contenido temático. Pudimos concluir que la organización estratégica en hipo-grupo es adoptada por los dirigentes que desean conceder a cada uno de los sucesores las mismas oportunidades para realizar los objetivos personales de estos últimos. La igualdad entre los sucesores permite evitar los conflictos internos que pueden pesar sobre la perpetuidad de la empresa. La elección de la actividad de la unidad recientemente creada depende del perfil del sucesor. Dado que los sucesores tienen perfiles diferentes, el desarrollo en hipo-grupo se conduce por medio de la diversificación.

\section{ZUSAMMENFASSUNG}

Die Interaktionen in einer Familie beeinflussen das Verhalten und die Wahrnehmung der Familienmitglieder und bestimmen die eigentliche Familienstruktur (Todd, 1983). Die Vielfalt solcher Strukturen kann direkt Einfluss auf die Strategie von Familienunternehmen haben. Die Entwicklung von Familienunternehmen, namentlich von kleinen, legt die Idee nahe, mit der Kreation von Untergruppen eine Wachstumsstrategie zu verfolgen. Ziel der Arbeit ist es, den Einfluss der Familienstruktur auf die Familienunternehmen, die in Subgruppen organisiert sind, zu untersuchen.

Die nötigen Informationen werden durch halb strukturierte Interviews bei Eigentümer-Geschäftsführern von Familien-Subunternehmen gesammelt und inhaltsanalytisch untersucht. Die Auswertung zeigt auf, dass die Aufteilung in Subunternehmen angewandt wird, um allen Nachfolgern die Möglichkeit zu bieten, ihre Ziele zu verfolgen. Diese Lösung vermindert das Aufkommen von internen Konflikten und verbessert den Fortbestand des Unternehmens. Die Schaffung der Teilbetriebe orientiert sich an den Eigenschaften der Nachfolger. Durch die Heterogenität dieser wird eine Diversifikation erzielt.

\section{Introduction}

La dimension familiale est particulièrement attractive pour comprendre comment le concours des considérations économiques et non économiques affecte les décisions stratégiques des entreprises familiales (Chrisman, Chua et Steier, 2003).

Pour les anthropologues de la famille, notamment Todd (1983), comprendre le management de l'entreprise familiale requiert la compréhension des relations sociales au sein de la famille. Inconsciemment mais inévitablement, chaque génération absorbe les valeurs parentales qui définissent les 
relations humaines élémentaires (entre parents et enfants). Ainsi, le modèle libéral insiste sur l'indépendance individuelle. Si le patrimoine familial est divisé d'une façon équitable entre tous les enfants, ce sera l'expression d'une relation égalitaire. $\mathrm{Si}$, au contraire, le système d'héritage se base sur l'indivisibilité de la propriété (un seul héritier), c'est alors un système inégalitaire.

En Tunisie, $84 \%$ des entreprises sont familiales. Sfax, à elle seule, dispose de plus de $25 \%$ de ce tissu entrepreneurial (Banque mondiale, 2000). Les travaux des chercheurs tunisiens illustrent bien le rôle de la famille comme un capital social catalyseur de développement (Basly, 2005; Chelly, 2006; Nabli, 2009). En Tunisie et à Sfax en particulier, les relations de sang fonctionnent comme supports de solidarité et de domination sociale (Zghal, 1994). Le lien de fraternité l'emporte sur tous les autres et débouche sur un système communautaire qui, dans les faits, permet le recyclage continuel du patrimoine au sein de la famille.

En fait, chaque membre est supposé travailler au sein de l'entreprise familiale mais il lui revient de fournir l'effort de tailler un territoire d'indépendance relative (Sharma et Manikutty, 2003). Il en résulte que tous les membres, traités équitablement, sont présumés rester sous l'autorité du père. Cependant, ils ont besoin d'un minimum d'indépendance que la diversification des activités, dans différents secteurs et par la création de nouvelles unités familiales, permet d'assurer dans un tel contexte.

Dans ce sens, Kellermanns et Eddleston (2006) critiquent la littérature relative à l'entreprise familiale. Ils estiment que celle-ci néglige l'investissement dans de nouveaux domaines d'activité alors qu'elle considère que l'entreprise familiale évite la prise de risque, résiste au changement et est fixée sur le maintien du statu quo. Ces auteurs reconnaissent le besoin de l'entreprise familiale de moderniser et d'intégrer de nouveaux marchés afin de survivre d'une génération à une autre. Ils soulignent l'importance de la création de nouvelles unités quant à la viabilité à long terme de ce type d'entreprise.

De ce fait, la création de nouvelles entreprises, dans le cadre d'une stratégie de développement de l'entreprise familiale, trouve ses origines dans la structure de la famille plutôt que dans la génération de revenus. Selon Granovetter (2005), ce type de structure est un ensemble d'entreprises reliées d'une façon formelle ou informelle. En matière de petite entreprise (PE) et de toute petite entreprise (TPE), Julien et Marchesnay (1987) adoptent la notion d'hypogroupe faisant ainsi référence aux caractéristiques de groupe dont il tire sa structure et à celles de l'hypofirme de point de vue de la taille de ses composantes. 
L'objectif de ce travail de recherche est d'étudier l'impact de la structure de la famille sur le développement de l'entreprise familiale en hypogroupe. Pour y parvenir, nous allons tout d'abord présenter les objectifs de l'entreprise familiale, puis définir la structure de la famille en tant que déterminant du développement de celle-ci pour ensuite décrire en quoi consiste l'organisation stratégique en hypogroupe. Enfin, après avoir exposé la méthodologie de recherche, nous rapporterons les résultats des analyses.

\section{Les objectifs de l'entreprise familiale}

Les recherches concernant l'entreprise familiale indiquent que les objectifs familiaux sont souvent des facteurs déterminants pour les décisions stratégiques puisque ce sont des facteurs qui influencent la dynamique organisationnelle de celle-ci (Dyer, 2003). Il est à noter que les objectifs de l'entreprise familiale sont multiples et complexes (Dunn, 1996). Ils sont, en plus, très différents de ceux des entreprises non familiales et publiques et dépassent l'objectif de maximisation de la valeur de l'entreprise (Daily et Dollinger, 1992). Dans la définition de l'entreprise familiale, on relève des objectifs communs à toutes les entreprises de ce type, soit le maintien du contrôle de la propriété et du management et la pérennité. Ces deux objectifs prioritaires font qu'une entreprise familiale se méfie particulièrement de la croissance par peur de perdre le contrôle de l'entreprise et, par conséquent, mettre en péril sa pérennité.

\subsection{Le maintien du contrôle de la propriété et du management}

La séparation entre la propriété et le contrôle managérial est une caractéristique fondamentale des entreprises capitalistes. Cette séparation est une source de conflits qui se traduit par des problèmes d'agence qui dépendent du degré de divergences entre les objectifs des dirigeants et ceux des actionnaires. Par ailleurs, la notion de contrôle familial intègre trois principales dimensions, liées aux fonctions de propriété, de contrôle et de direction pouvant être exercées par les membres de la (des) famille(s) actionnaire(s) (Allouche, Amann et Garaudel, 2006).

La préférence de la famille de maintenir un contrôle personnel s'explique par un système de contrôle peu formalisé où la propriété et le management sont réunis (Daily et Dollinger,1992). Néanmoins, même si l'objectif de préserver le contrôle de la propriété ou du management est au cœur de la notion d'entreprise familiale, le fait de trouver un moyen de se développer, tout en préservant la nature familiale de l'entreprise, demeure problématique. 
Ainsi, la structure organisationnelle devient un outil permettant de résoudre les problèmes spécifiques au maintien de la nature familiale de l'entreprise puisque cette structure précise le cadre explicite des relations entre acteurs et la répartition des rôles, de prise de décisions, d'orientation stratégique et de contrôle. Cependant, si l'entreprise familiale introduit un formalisme excessif, cela peut conduire à diluer le contrôle et, dans ce cas, cette structure ne résout pas toutes les difficultés (Barrédy, 2002).

\subsection{La pérennité}

La pérennité de l'entreprise suppose qu'elle continue d'être la propriété de la famille et dirigée par ses membres (Mignon, 2000). Le principal problème qui se pose au dirigeant est de décider comment il va procéder pour assurer la pérennité de l'entreprise par sa transmission à la génération suivante. Gaultier (1990) explique que, très souvent, des entreprises familiales sont menacées de disparaître ou d'éclater sous la pression des héritiers qui poursuivent des buts différents. Étape majeure dans la survie de l'entreprise, la transmission reste un objectif de premier ordre car toutes les entreprises ne réussissent pas leur transmission (Chua, Chrisman et Sharma, 1999).

Le dirigeant souhaite la continuité de l'entreprise uniquement si elle reste dans le patrimoine familial. Il s'agit alors d'une transmission familiale (interne), dans laquelle le successeur est l'héritier (Defreyman, RichommeHuet et Paturel,2006). Cette transmission au sein de la famille par opposition à la transmission salariale ou à la cession à des tiers est alors l'objet de notre étude. Elle peut être définie comme «un processus dynamique durant lequel les rôles et les fonctions des deux principaux groupes d'acteurs, soit le prédécesseur et le successeur, évoluent de manière dépendante et imbriquée, cela dans le but ultime de transférer à un membre de la prochaine génération, d'une part, la direction de l'entreprise et, d'autre part, la propriété » (Cadieux et Lorrain, 2002, p. 5).

Ainsi, le plan de la transmission est un processus délibéré et formel qui facilite le transfert du contrôle managérial d'un membre de la famille à un autre (Sharma, 1997). De ce fait, les actions nécessaires à la transmission ne sont pas neutres dans le développement futur de l'entreprise. Celles qui seront menées parmi l'ensemble des choix possibles sont des éléments moteurs qui doivent concourir à terme à assurer la pérennité de l'entreprise. En fonction de la nature de la transmission qui aura été mise en place dans l'entreprise, des changements stratégiques et de nouvelles orientations stratégiques spécifiques à cette transmission prendront place (Haddadj et Andria, 2001). 


\section{La structure de la famille}

La structure de la famille est le système d'interactions au sein de la famille qui influence le comportement de chaque membre et sa perception de la réalité (Todd, 1983). Dans ce qui suit, nous allons présenter la typologie de la structure de la famille et le lien pouvant exister entre cette dernière et le développement de l'entreprise familiale en hypogroupe.

\subsection{La typologie de la structure de la famille}

Todd (1983) élabore une typologie des structures familiales montrant comment certaines d'entre elles sont régies de façon autoritaire par un patriarche alors que d'autres sont très libertaires et laissent beaucoup d'autonomie aux enfants. L'application des deux principes d'autorité et d'égalité, pouvant chacun prendre deux valeurs opposées, engendre une typologie comptant quatre catégories:

- La famille nucléaire absolu est libérale et inégalitaire. Les membres de la famille sont indépendants et orientés vers l'accomplissement personnel. Dans les rapports entre les enfants, la grande différence apparaît au moment de l'héritage. Les enfants ne sont absolument pas égaux. Il revient aux parents de répartir leur héritage, leurs biens, de façon complètement libre entre leurs enfants.

- La famille nucléaire égalitaire est caractérisée par les concepts de liberté et d'égalité. Le passage à cet état d'indépendance suppose un certain apprentissage de la liberté pour les enfants. À aucun moment dans ce type familial, on n'a d'enfants, adultes et mariés, qui vivent toujours sous l'autorité de leurs parents. Dans ce type nucléaire et égalitaire, lors du décès des parents, l'héritage est partagé de façon absolument égalitaire entre tous les enfants.

- La famille autoritaire incarne les valeurs d'inégalité et d'autorité. L'ultime autorité est accordée au père et un seul héritier est désigné. Un des enfants, généralement l'aîné des enfants (mais ce n'est pas toujours ainsi), est donc choisi comme héritier. Il passe à un statut d'adulte, tout en restant sous l'autorité des parents, en tout cas sous l'autorité du père. Les autres enfants, garçons ou filles, sont éjectés de la famille. L'entrée dans la vie adulte n'implique pas l'indépendance par rapport aux parents.

- La famille communautaire est caractérisée par les valeurs d'égalité et d'autorité. L'autorité est la valeur fondamentale de ce système familial, puisque tous les enfants restent sous l'autorité du père. Le 
fait de ne pas distinguer d'héritier unique induit un raisonnement plus égalitaire. Il s'agit d'une valeur très forte d'égalité, car il existe un principe de symétrie entre les enfants qui sont tous au même niveau. D'ailleurs, lorsque le père décède, l'héritage est partagé de façon absolument égalitaire.

Todd (1983) analyse les structures familiales en distinguant les rapports entre parents et enfants selon qu'ils sont de type libéral ou de type autoritaire et les rapports entre enfants selon qu'ils sont égalitaires ou inégalitaires. Si l'on croise ces deux variables, on obtient quatre types familiaux fondamentaux.

TABLEAU 1

La typologie de la structure de la famille

\begin{tabular}{ccc}
\hline $\begin{array}{l}\text { Relations } \\
\text { enfants-enfants }\end{array}$ & Autorité & Liberté \\
\hline $\begin{array}{c}\hat{E} \text { galité } \\
\text { Inégalité }\end{array}$ & $\begin{array}{c}\text { Communautaire } \\
\text { enfants }\end{array}$ & $\begin{array}{c}\text { Nucléaire égalitaire } \\
\text { Nucléaire absolu }\end{array}$ \\
\hline
\end{tabular}

Notre étude part du postulat général selon lequel la famille à Sfax (Tunisie) est du type communautaire (égalitaire et autoritaire). Cette structure est un facteur important en matière de principes et d'enjeux d'organisation et de coordination humaine, dans la mesure où elle est la première instance d'organisation socioculturelle (Nabli, 2009).

\subsection{La structure de la famille et le développement en hypogroupe}

Dans bien des cas, le principal problème qui se pose au fondateur est comment assurer la pérennité de l'entreprise par sa transmission à la génération suivante. Certains fondateurs recherchent un héritier unique qui pourra maintenir la concentration du pouvoir entre les mains du dirigeant-propriétaire de l'entreprise. Toutefois, un plus grand nombre d'entre eux conçoivent l'entreprise comme un patrimoine collectif qu'ils partagent entre les membres de la famille (Ward, 2005). Cependant, renoncer à l'exercice du pouvoir serait, selon Brunner (1975), la contrainte absolue qui mettrait un obstacle définitif aux projets de développement dans lesquels l'exercice du pouvoir serait aliéné.

Hormis les enjeux du pouvoir, la centralisation n'est pas propice à l'intégration d'un autre domaine d'activité et d'un autre dirigeant, car cela pose des problèmes organisationnels. La croissance par la diversification, en agrandissant l'organisation et le nombre des niveaux hiérarchiques, la 
complexifie inévitablement. À terme, la centralisation cède la place à une plus grande délégation, ce qui pourrait être difficile à accepter et s'avère peu compatible avec l'idéologie dominante (Kalika, 1988).

La structure de la famille permet de tirer profit de l'expérience accumulée et de l'expérience du passé et sera utilisée probablement comme une aide à la décision. Les différents membres de la famille dirigent différentes entreprises indépendantes mais qui sont toujours liées et font partie de la même propriété familiale. En introduisant la notion de structure familiale dans notre analyse du développement des entreprises familiales, nous considérons que cette stratégie est délibérément mise en œuvre pour maintenir l'unité familiale et, comme l'a annoncé Mintzberg (1982), l'organisation de l'entreprise résulte de l'histoire de celle-ci et des rapports de pouvoir.

Favorisée par l'égalité qui définit la structure de la famille tunisienne, la recherche du bien-être pousse le dirigeant-propriétaire à créer de nouvelles unités afin de répondre aux objectifs de ces membres tout en étant égalitaire. Ainsi, le développement en hypogroupe permet de résoudre les conflits inhérents à la divergence entre les priorités des différents membres, d'offrir l'indépendance à chacun et d'assurer les mêmes avantages et les mêmes opportunités de participation au contrôle du management familial.

Ainsi, nous estimons que les nouvelles unités sont créées en réponse à une structure familiale du type communautaire. Ce type de développement offre la possibilité de faire cohabiter plusieurs membres familiaux sous le signe de l'égalité en accordant à chacun le droit de créer sa propre unité familiale et de mener la direction d'une entreprise indépendante. Cette stratégie de développement préserve les valeurs familiales et permet au patriarche de continuer d'exercer l'ultime autorité puisque le contrôle de l'unité d'origine lui sera réservé. À partir de là, notre proposition est la suivante:

Proposition 1. La structure de la famille communautaire affecte le développement de l'entreprise familiale par la création de nouvelles unités: la répartition des responsabilités de gestion est égalitaire entre les enfants d'une même génération.

\section{La forme d'hypogroupe}

Il convient d'apprécier la pertinence des formes d'organisation au regard des spécificités de chaque entreprise, lesquelles tiennent à la stratégie qu'elle développe et à son propre stade de développement, plutôt que d'y adhérer de façon aveugle. 
Le développement des entreprises familiales, notamment de petite taille, introduit l'idée d'une forme d'organisation susceptible de permettre la mise en œuvre de stratégies de croissance. Cette croissance s'est également accompagnée du développement de structure comme les groupes (Huppert, 1981; Debray, 1997; Leyronas, 2004). Cette structure se justifie lorsqu'il faut gérer plusieurs dimensions stratégiques interdépendantes et réputées d'importances équivalentes que l'on s'interdit de subordonner les unes aux autres (Crémadez, 2004).

Relativement aux travaux de Debray (1997), il existe des petits groupes qui ont fait le choix de cette structure pour conserver les avantages liés à la petite taille et pour lesquels il n'est pas envisageable de croître autrement que par l'adjonction de nouvelles petites entreprises. Julien et Marchesnay (1987) adoptent la notion d'hypogroupe faisant ainsi référence aux caractéristiques de groupe dont il tire sa structure et à celles de l'hypofirme de point de vue de la taille de ses composantes. Par ailleurs, le dirigeant de l'hypogroupe familial est mû par une logique «stock» d'accumulation patrimoniale et un souci de pérennisation de l'affaire dans l'objectif de la transmettre à ses héritiers.

L'hypogroupe est une structure de groupe dont les composantes sont des petites, voire toutes petites entreprises, unies entre elles par des relations financières. Ces relations donnent à l'une d'entre elles (la holding) le pouvoir de décision sur les autres. Quelle que soit la combinaison utilisée dans la structure de propriété du capital, elle confère au dirigeant-propriétaire une place centrale et le pouvoir de décision final concernant la gestion stratégique de l'hypogroupe (Debray, 1997).

\subsection{Le développement par la voie de la diversification}

L'hypogroupe est considéré comme une forme d'organisation spécifique spécialement soutenue pour faciliter le processus de diversification de l'entreprise familiale (Iacobucci, 2002). Le point de départ de cette analyse est le fait que l'hypogroupe est le résultat d'un processus de développement mis en œuvre par la création de plusieurs entreprises. L'hypogroupe familial résulte donc du choix du dirigeant d'une stratégie de diversification (Rosa, 1998; Rosa et Scott, 1999). Cela implique que cet hypogroupe n'est pas simplement une structure organisationnelle mais aussi un processus de développement lié à la stratégie de diversification.

Concernant la voie de développement choisie par les groupes familiaux, Boissin (2000) estime «qu'à partir d'une position extérieure éloignée de l'entreprise, le chercheur conclurait à une opération d'expansion horizontale, c'est-à-dire une spécialisation du groupe dans son domaine d'activité le plus 
large. Mais en fait, cette stratégie de développement émerge d'opportunités et se traduit par un processus de diversification induit et incrémental du portefeuille d'activités stratégiques en matière de technologies et services clients» (p.5). Le groupe autorise une répartition des risques filiale par filiale. L'indépendance juridique des filiales permet de diminuer le risque financier provenant de la diversification des activités.

Par conséquent, la stratégie de constitution de l'hypogroupe est de faire travailler les filières entre elles afin de produire des synergies industrielles ou commerciales, ce qui ne se retrouve pas nécessairement dans les grands groupes (Debray et Leyronas, 1996). L'étude de Iacobucci et Rosa (2005) des entreprises italiennes révèle que l'objectif du développement des entreprises familiales en hypogroupe est de sécuriser le contrôle de certaines phases de la filiale de production et de développer, en même temps, le domaine d'activité d'origine. Ils montrent que l'intégration verticale concerne le quart des nouvelles entreprises créées. D'où la proposition suivante:

Proposition 2. Le développement par la voie de la diversification conduit au choix de l'organisation stratégique en hypogroupe.

\subsection{L'hypogroupe: une manœuvre de contrôle}

Le développement en hypogroupe est une stratégie de développement d'activités autour d'un métier permettant un meilleur contrôle de l'hypogroupe sur ses approvisionnements et ses débouchés et favorisant un accroissement de son pouvoir de marché. C'est aussi une stratégie de minimisation de certains risques issus à la fois de la petite taille (soumission à l'environnement) mais aussi attachés à la grande dimension (la perte de contrôle et d'indépendance, la bureaucratie, etc.). Ce développement est justifié par la recherche d'un plus haut degré de diversification des activités et par la possibilité de bénéficier de la responsabilité limitée des différentes entreprises afin de réduire le risque associé à ces activités.

En fait, la volonté de préserver l'indépendance de l'entreprise et le contrôle familial constitue l'une des principales motivations guidant le choix de l'entreprise familiale et conditionne ses options stratégiques (Allouche, Amann et Garaudel, 2006). L'hypogroupe est, dans ce cas, une forme de développement intermédiaire qui, sans croître en taille, permet de maintenir le contrôle familial de l'entreprise et d'éviter les risques de dépendance des partenaires (autonomie/indépendance). Par conséquent, les facteurs favorisant le choix de la structure d'hypogroupe, les avantages que procure 
cette forme pour l'entreprise familiale en plus des attributs spécifiques à la petite taille incitent le dirigeant de l'entreprise familiale à développer son entreprise sous forme d'hypogroupe.

Ainsi, nous expliquons la logique du développement des entreprises familiales en hypogroupes par le schéma suivant (figure 1):

\section{FIGURE 1}

\section{L'impact de la structure de la famille sur le développement en hypogroupe}

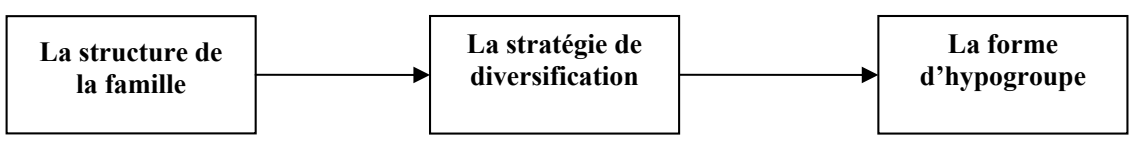

En conclusion, la structure de la famille mène à la création de nouvelles unités pour accorder à chaque membre de la famille les mêmes responsabilités et la même indépendance. Par ailleurs, les objectifs de l'entreprise familiale, notamment le maintien du contrôle à travers la petite taille, contribuent au choix de la forme d'hypogroupe afin de structurer ce développement. L'entreprise familiale se trouve alors engagée dans une stratégie de diversification résultant de l'intégration de nouveaux secteurs d'activité lors de la création des nouvelles unités.

\section{Les choix méthodologiques}

L'hétérogénéité des PME familiales et les diverses formes qu'elles peuvent prendre a considérablement complexifié notre tâche de trouver des entreprises qui correspondent aux critères de notre sujet de recherche, à savoir des groupes de petites entreprises familiales. De plus, l'ambiguïté des concepts étudiés et la volonté de comprendre la logique du développement des entreprises familiales en hypogroupes, difficilement quantifiable, nous ont incités à adopter une démarche qualitative.

En effet, la démarche qualitative offre des avantages pour des recherches internes et dynamiques des organisations dans lesquelles le comportement et les interprétations sont repérables par les jugements des individus. L'axe d'enrichissement de cette démarche consiste à adopter une approche qualitative inductive afin d'identifier les traits du contexte qui paraissent les plus pertinents pour l'étude. Cela favorise l'émergence d'explications à partir de données empiriques plutôt que d'insérer les données dans un cadre préalablement établi et qui n'existe pratiquement pas pour notre sujet. 
À l'instar de Marchesnay (1993), pour ce type de recherche, plusieurs auteurs prônent le développement des approches expérimentales, au cas par cas, plutôt que de rechercher des grandes synthèses prescriptives, déterministes et supposées valables pour tous les problèmes à traiter. Pour ce faire, la méthode des cas est un mode d'observation précis de thèmes préalablement définis par le questionnement (Yin, 1994). C'est une technique particulière de collecte, de mise en forme et de traitement de l'information qui cherche à rendre compte des explications des dirigeants-propriétaires de leurs choix de développement sous forme d'hypogroupes (Mzid et Mezghani, 2008). Certains phénomènes ne peuvent se comprendre que par une analyse fine comme celle que permet l'étude de cas (Deslauriers et Kérisit, 1997). Les dirigeants vont alors décrire les situations liées à la structure familiale, notamment l'égalité, qui ont conduit à la création de nouvelles unités.

La principale limite de l'approche qualitative est qu'elle s'inscrit dans une démarche d'étude d'un contexte particulier. De ce fait, le recours à l'analyse de plusieurs contextes nous permet d'accroître la validité externe d'une recherche qualitative selon une logique de réplication (Thiétart et al., 2004). Cependant, l'approche qualitative offre plus de garantie sur la validité interne des résultats.

\subsection{La sélection des cas}

La sélection des cas de l'étude est en revanche plus complexe en raison du nombre de cas à traiter. Toutefois, Eisenhardt (1989) remarque que la plupart des recherches de ce type incluent un nombre compris entre quatre et dix cas. Les exemples de recherches fournis par Yin (1994), qui préconisent une logique de réplication successive pour autoriser la généralisation de l'étendue, n'excèdent pas les 12 cas.

La grande difficulté que pose le choix des hypogroupes familiaux est liée au fait qu'en Tunisie il n'existe pas de statut juridique reconnu de l'entreprise familiale. Pour cette raison, nous avons eu recours à l'API (Agence de promotion de l'industrie) afin de sélectionner les groupes familiaux mais les hypogroupes ne sont recensés par aucun organisme.

Ainsi, les hypogroupes, objets d'analyse, ont été sélectionnés sur la base des critères liés à la fois à la définition que nous avons adoptée de l'entreprise familiale, à des critères spécifiques à la petite entreprise et aux caractéristiques du groupe. C'est à travers des dialogues avec des dirigeants d'entreprises, l'exploitation des réseaux et des entretiens que nous avons pu constituer un échantillon de cinq hypogroupes de la région de Sfax (sud de la Tunisie). 
La difficulté de repérer les hypogroupes, la prise de rendez-vous avec les dirigeants très occupés par la gestion de l'hypogroupe et la durée nécessaire pour un entretien en profondeur présentent certaines contraintes. Ainsi, il n'y a pas eu de sélection aléatoire mais tous les dirigeants-propriétaires des hypogroupes sont systématiquement interrogés selon la même démarche.

\subsection{La collecte des données}

La conduite de l'entretien varie en fonction des hypogroupes considérés, c'est-àdire que nous avons procédé d'une manière appelée par Bourdieu (1994) «improvisation réglée»: une improvisation, parce que chaque entretien est une situation singulière susceptible de produire des effets de connaissance particuliers; réglée, car, pour produire ces effets de connaissance, l'entretien demande un certain nombre d'ajustements qui constituent à proprement parler la technique de l'entretien (Blanchet et Gotman, 1992).

L'information est alors collectée par le biais d'un entretien semi-directif qui se prête bien à la nature inductive de cet aspect de la recherche. Nous avons alors interviewé, pour chaque cas d'hypogroupe, deux dirigeantspropriétaires de deux générations différentes et, lorsque c'est possible, le père fondateur et son successeur. Chacune des personnes interviewées dirige une unité indépendante de l'hypogroupe. Cet entretien se déroule à partir d'un guide défini au préalable et qui liste les thèmes qui doivent être abordés dans le courant de l'entretien. Notre objectif étant de repérer l'impact de la structure de la famille sur le développement des entreprises familiales en hypogroupes, les thèmes abordés apportent des réponses pour trois principaux axes d'interrogation:

- Qui dirige les différentes entreprises?

- Qui seront les dirigeants du futur?

- Quels sont les objectifs visés par le développement en hypogroupe?

Par ailleurs, dans le contexte des petites entreprises familiales tunisiennes étudiées, force a été de constater que celles-ci génèrent très peu de documentation sur elles-mêmes ou sur leur histoire, ou bien que ces documents ne sont pas d'ordre public. Par conséquent, les entretiens ont fourni le principal matériau permettant de développer les cas. Ainsi, nous invitons les interrogés à se prononcer sur ces thématiques s'ils ne le faisaient pas eux-mêmes spontanément. Nous avons également retenu les faits dont l'interviewé (le dirigeant-propriétaire) nous faisait part et auxquels nous n'avions pas pensé auparavant. 


\subsection{Le traitement des données}

Pour mieux connaître les déterminants du développement des entreprises familiales en hypogroupes, nous procédons par une analyse du contenu thématique. Ce type d'analyse fait en quelque sorte disparaître la singularité du discours et découpe transversalement ce qui, d'un entretien à l'autre, se réfère au même thème. Elle ignore ainsi la cohérence singulière de l'entretien et cherche une cohérence thématique interentretiens (Blanchet et Gotman, 1992).

Comme le préconisent Miles et Huberman (2003), nous avons défini un projet de codage précis, qui a établi au préalable une liste de codes que nous avons attribués aux segments de texte sélectionnés. Plus qu'une approche purement descriptive, nous avons opté pour un codage par induction afin de dégager des tendances susceptibles d'expliquer le développement en hypogroupe (Mzid et Mezghani, 2008). Lorsqu'un segment de texte semblait pouvoir faire l'objet d'un codage multiple, c'est-à-dire qu'il représentait simultanément plusieurs thèmes, nous avons opté pour un codage multiple afin d'éviter de «forcer» les données vers une variable particulière (Vas, 2005). Dans le souci de préserver l'anonymat de nos interlocuteurs et de leurs entreprises, nous désignons les cas par des numéros.

\section{Les résultats de l'étude de la structure de la famille}

Comme nous ne pensons pas pouvoir accéder d'une façon objective à la réalité, nous adoptons la position du chercheur constructiviste qui, selon Rispal (2002), se concentre sur des représentations de la réalité afin de comprendre la réalité du phénomène à travers les interprétations des acteurs ou essentiellement de la construire.

Dans ce qui suit, nous présentons les résultats de l'analyse intra-cas en décrivant la relation entre la structure de la famille et le développement de l'entreprise familiale en hypogroupe. Ensuite, nous discutons l'analyse inter-cas et présentons une synthèse de l'impact de la structure de la famille sur l'émergence des hypogroupes familiaux.

\subsection{L'analyse intra-cas de la structure de la famille}

Les cas étudiés sont présentés selon les thèmes abordés, à savoir qui dirige les différentes unités, qui seront les dirigeants du futur et quels sont les objectifs visés à travers le développement en hypogroupe, et ce, après avoir avancé une présentation générale de chaque cas. 
Cas 1. Cet hypogroupe a été fondé par deux frères et l'épouse de leur troisième frère. Chacun des fondateurs et leurs successeurs dirigent les six unités qui sont présentées ci-dessous. Nous avons pu interviewer le fondateur (2) et son fils unique, le successeur (3).

TABLEAU 2

Présentation des unités de l'hypogroupe 1

\begin{tabular}{lcccc}
\hline & $\begin{array}{c}\text { Date de } \\
\text { création }\end{array}$ & Effectif & Activité & Dirigeant \\
\hline $\begin{array}{l}\text { Unité 1 } \\
\text { (entreprise } \\
\text { d'origine) }\end{array}$ & 1978 & 25 & Commerciale & Fondateur 1 \\
\hline Unité 2 & 1984 & 50 & Fonderie de fonte & Fondateur 2 \\
\hline Unité 3 & 1988 & 10 & Agriculture & Fondatrice \\
\hline Unité 4 & 1995 & 39 & Industrie textile & $\begin{array}{c}\text { Successeur 1 } \\
\text { (fils du fondateur 1) }\end{array}$ \\
\hline Unité 5 & 2002 & 13 & $\begin{array}{c}\text { Ordinateurs et } \\
\text { autres équipements } \\
\text { informatiques. }\end{array}$ & $\begin{array}{c}\text { Successeur 2 } \\
\text { (fils du fondateur 1) }\end{array}$ \\
\hline Unité 6 & 2003 & 7 & Services & $\begin{array}{c}\text { Successeur 3 } \\
\text { (fils du fondateur 2) }\end{array}$ \\
\hline
\end{tabular}

Tous les membres de cette famille bénéficient des mêmes avantages et des mêmes parts de capital. Afin d'assumer cette structure égalitaire, il fallait traiter uniformément les successeurs et éviter parallèlement les conflits entre les membres d'une même génération. Comme les successeurs ne pouvaient pas tous intégrer l'entreprise d'origine à cause de leur nombre, les fondateurs ont envisagé la création d'une unité indépendante dont les activités touchent aux compétences du successeur considéré. Étant égalitaire, le prédécesseur a répété la même procédure pour tous les successeurs qui se trouvent dans la même situation donnant ainsi naissance à l'hypogroupe.

Il est à noter que le fondateur (2) exprime une plus grande égalité puisqu'il est responsable de cette égalité entre successeurs. Cependant, le successeur (3) confirme une certaine autorité de ce fondateur: il l'a forcé à travailler avec lui et lui a caché son passeport, l'obligeant ainsi à intégrer l'entreprise familiale. Même en tant que dirigeant, le père obligeait son fils à respecter les horaires de travail. D'ailleurs, c'est pour cette raison que le successeur (3) a du mal à collaborer avec son père et a préféré s'éloigner un peu de lui pour jouir d'une certaine autonomie en sollicitant la direction d'une unité autonome. Lorsque le fondateur (2) s'est rendu compte que cette attitude ne faisait qu'accentuer les conflits, il lui a créé une nouvelle unité indépendante. Cette manœuvre assure au successeur (3) une certaine 
indépendance, mais elle permet au prédécesseur de contrôler les mouvements de son fils et ses aptitudes de gestion. Néanmoins, l'incompétence des fondateurs eu égard aux activités de leurs successeurs restreint leur accès à ces domaines. L'autorité est de ce fait réduite au profit d'une plus grande autonomie des successeurs.

Cas 2. Cet hypogroupe a été développé par les successeurs à partir d'une activité d'origine fondée par leur père. Il est à noter que la direction des unités de cet hypogroupe est assurée par deux successeurs seulement parmi les trois. En fait, le troisième successeur possède les mêmes parts de capital mais étant médecin, il ne peut pas exercer l'activité de gestion. Nous avons alors interviewé ces deux directeurs-propriétaires. Cet hypogroupe est présenté comme suit.

TABLEAU 3

Présentation des unités de l'hypogroupe 2

\begin{tabular}{lccccc}
\hline & $\begin{array}{c}\text { Date de } \\
\text { création }\end{array}$ & Effectif & Activité & Produit & Dirigeant \\
\hline $\begin{array}{l}\text { Unité 1 } \\
\text { (entreprise } \\
\text { d'origine) }\end{array}$ & 1963 & 47 & Industrie textile & $\begin{array}{c}\text { Vêtements } \\
\text { de travail }\end{array}$ & $\begin{array}{c}\text { Fondateur/ } \\
\text { successeur 1 }\end{array}$ \\
\hline Unité 2 & $\begin{array}{c}1963 \\
(1998)\end{array}$ & 45 & Industrie textile & $\begin{array}{c}\text { Vêtements } \\
\text { modernes }\end{array}$ & $\begin{array}{c}\text { Successeur 1 (fils } \\
\text { aîné du fondateur) }\end{array}$ \\
\hline Unité 3 & 1997 & 20 & Agroalimentaire & Aviculture & $\begin{array}{c}\text { Successeur 2 } \\
\text { (fils cadet) }\end{array}$ \\
\hline Unité 4 & 1999 & 20 & Agroalimentaire & Huilerie & Successeur 2 \\
\hline Unité 5 & 2000 & 10 & Services & $\begin{array}{c}\text { Promotion } \\
\text { immobilière }\end{array}$ & Successeur 1 et 2 \\
\hline
\end{tabular}

Cette famille est du type communautaire. Cependant, les successeurs (1) et (2) estiment que, d'un côté, l'égalité est un facteur déterminant du succès du processus de transmission, dès lors, de la pérennité. D'un autre côté, l'autorité du père réduit l'indépendance et l'autonomie d'action des successeurs, ce qui peut être source de tension et peut engendrer des conflits entre les différentes générations. Cela peut conduire à l'échec du processus de transmission, mettant en péril la pérennité de l'entreprise familiale.

Ainsi, pour assurer cette pérennité,il est nécessaire d'accorder, de façon égalitaire, les responsabilités de gestion aux successeurs. En effet, l'inégalité est source de conflits entre successeurs à cause des sentiments de jalousie que suscite le fait d'avoir son frère comme supérieur hiérarchique. Il faut dès lors créer de nouvelles unités si la taille de l'entreprise ou la nature de son activité ne permet pas d'assumer cette égalité. 
En outre, l'autorité du père est contournée par la création de nouvelles unités afin de s'assurer de l'indépendance des successeurs. En fait, le successeur (2), avec un peu de recul, estime qu'en fin de compte il n'avait pas choisi de travailler dans l'entreprise familiale et que ce n'est qu'une conséquence de l'autorité de son père. Ainsi, la création de nouvelles unités indépendantes opérant dans des secteurs non liés à l'activité d'origine permet d'acquérir une certaine autonomie.

Cas 3. Cet hypogroupe en est à sa troisième génération. Le prédécesseur, ayant assuré la pérennité de l'entreprise familiale pour sa deuxième génération, est décédé. Ce sont ses enfants qui ont pris la relève. Cependant, la différence d'âge entre le premier et les autres successeurs est un peu grande. De ce fait, la relation entre ces membres se rapproche de celle reliant deux générations distinctes. Nous avons pu conduire les entretiens avec le successeur (1), l'aîné, et le successeur (2). Par ailleurs, cet hypogroupe œuvre dans les domaines suivants tels que décrits dans le tableau 4.

\section{TABLEAU 4}

\section{Présentation des unités de l'hypogroupe 3}

\begin{tabular}{lccccc}
\hline & $\begin{array}{c}\text { Date de } \\
\text { création }\end{array}$ & Effectif & Activité & Produit & Dirigeant \\
\hline $\begin{array}{l}\text { Unité (1) } \\
\text { (entreprise } \\
\text { d'origine) }\end{array}$ & 1948 & 15 & Agroalimentaire & Huilerie & $\begin{array}{c}\text { Successeur 3 } \\
\text { (frère cadet) }\end{array}$ \\
\hline Unité 2 & 1971 & 35 & $\begin{array}{c}\text { Industrie } \\
\text { mécanique }\end{array}$ & $\begin{array}{c}\text { Matériel } \\
\text { agricole }\end{array}$ & $\begin{array}{c}\text { Successeur 4 } \\
\text { (cousin) }\end{array}$ \\
\hline Unité 3 & 1998 & 12 & Commerciale & Automobiles & $\begin{array}{c}\text { Successeur 1 } \\
\text { (frère aîné) }\end{array}$ \\
\hline Unité 4 & 2005 & 8 & Commerciale & Automobiles & $\begin{array}{c}\text { Successeur 2 } \\
\text { (second frère) }\end{array}$ \\
\hline
\end{tabular}

Le capital est réparti d'une façon égalitaire entre les successeurs avec une petite majoration pour le dirigeant de l'unité en titre de motivation; cette égalité évite aussi les conflits entre enfants. Le successeur (1) estime que ce sont les avantages qui créent les tensions et les conflits entre les membres de la famille. Il pense que seule l'égalité permettrait d'assurer la pérennité de l'entreprise familiale sans susciter de conflits. Il affirme aussi que la quête d'égalité était à l'origine de la multiplication des entreprises. En fait, c'est le père qui avait implanté cet état d'esprit et qui avait insisté sur l'union familiale et sur le fait que les successeurs devaient être associés dans toutes les activités.

Quant au successeur (2), il estime que l'importance accordée à l'égalité pousse à multiplier les unités. Ainsi, les successeurs ont tous les mêmes 
responsabilités et bénéficient de la même autonomie de gestion. Désormais, chaque successeur dirige une unité indépendante. Le capital de chaque unité est divisé en parts égales. Le prédécesseur avait transmis le savoir-faire à ses enfants sans avoir recours à l'autorité; il ne les avait pas obligés à intégrer l'entreprise familiale. Ils l'ont fait de leur propre gré après que le successeur (1) eut choisi d'assumer la relève. Depuis, ce dernier joue le rôle du père sans être, lui non plus, autoritaire. Ce successeur est celui qui a le plus côtoyé son père. Il nous a affirmé que ce dernier les a toujours encouragés à diriger, à prendre les décisions d'une façon autonome, à agir sans crainte et à faire des erreurs.

Concernant le choix des activités, la stratégie de diversification n'a pas été recherchée. Ce n'est qu'une conséquence d'un ensemble d'actions mises en œuvre par opportunisme. L'objectif général de cette voie de développement est, en réalité, la diversification des risques. L'intégration de domaines non liés permet de compenser les aléas des différents secteurs.

Cas 4. Pour ce cas, nous avons eu un entretien avec le dirigeant fondateur et son fils le successeur (2). Cet hypogroupe est encore à sa première génération et ses membres ont une volonté manifeste de transmission. Il est présenté comme suit.

TABLEAU 5

Présentation des unités de l'hypogroupe 4

\begin{tabular}{lccccc}
\hline & $\begin{array}{c}\text { Date de } \\
\text { création }\end{array}$ & Effectif & Activité & Produit & Dirigeant \\
\hline $\begin{array}{l}\text { Unité } 1 \\
\text { (entreprise } \\
\text { d'origine) }\end{array}$ & 1972 & 49 & Agroalimentaire & $\begin{array}{c}\text { Poissons et autres } \\
\text { fruits de mer } \\
\text { congelés. }\end{array}$ & $\begin{array}{c}\text { Successeur 1 } \\
\text { (fils aîné) }\end{array}$ \\
\hline Unité 2 & 1997 & 25 & Agroalimentaire & Thon frais. & $\begin{array}{c}\text { Successeur 3 } \\
\text { (troisième fils) }\end{array}$ \\
\hline Unité 3 & 2000 & 20 & Agroalimentaire & $\begin{array}{c}\text { Conditionnement } \\
\text { de coquillage et } \\
\text { autres fruits de mer. }\end{array}$ & $\begin{array}{c}\text { Successeur 2 } \\
\text { (deuxième fils) }\end{array}$ \\
\hline Unité 4 & 2003 & 41 & Agroalimentaire & $\begin{array}{c}\text { Mollusques } \\
\text { surgelés. }\end{array}$ & $\begin{array}{c}\text { Successeure 4 } \\
\text { (fille) }\end{array}$ \\
\hline Unité 5 & 2004 & 15 & Agriculture & $\begin{array}{c}\text { Asperges sauvages } \\
\text { et champignons. }\end{array}$ & $\begin{array}{c}\text { Fondateur } \\
\text { (père) }\end{array}$ \\
\hline
\end{tabular}

Le fondateur a expliqué qu'il a quatre enfants dont une fille; cependant, tout au long de son discours, il ne fait pas cette distinction. Le capital et les responsabilités de management ont, alors, été répartis à égalité entre les trois frères et la sœur. La structure de cette famille propriétaire est donc de type égalitaire.

Selon le successeur, son père fondateur considère ses quatre enfants sur le même pied d'égalité où chacun dirige d'une façon indépendante une 
unité de l'hypogroupe. Ainsi, pour ce dirigeant-propriétaire, l'organisation stratégique d'hypogroupe assure l'égalité entre les successeurs. Chacun des successeurs a choisi lui-même, avec le consentement de ses frères, l'unité à diriger. Il ne s'agit pas de partager les entreprises. Les successeurs sont tous propriétaires à parts égales de toutes les unités de l'hypogroupe afin de ne pas dire par la suite que l'un des frères a eu l'entreprise la plus rentable ou l'entreprise la plus développée. Il s'agit du partage des responsabilités de gestion en faisant bénéficier chaque successeur de son autonomie et de la liberté d'action grâce à l'indépendance des unités de l'hypogroupe.

En ce qui concerne l'autorité, tout ce qui a été avancé par les deux dirigeants-propriétaires est en faveur de l'autonomie et de l'indépendance des successeurs. Le fondateur n'avait rien imposé à ses enfants mais il les a imprégnés dès leur jeune âge par sa logique patrimoniale. Il les a préparés à la relève et leur a transmis ses savoir-faire. Il s'est effectivement retiré pour s'occuper d'une activité agricole complètement éloignée des domaines dirigés par ses successeurs. Le domaine agricole est le passe-temps favori du prédécesseur. Le successeur pense que le développement de cette activité permet d'occuper son père pendant sa retraite pour qu'il ne sente pas le vide engendré par son désengagement de l'entreprise familiale d'origine.

Néanmoins, la décision prise au niveau des unités de l'hypogroupe est collégiale. Le successeur (2) confirme que c'est par respect que chaque dirigeant tient compte de l'avis de son père lors de la prise de décision et non par soumission à l'autorité. Il justifie cela par le fait qu'ils visent la pérennité et la performance de tout l'hypogroupe et non pas d'une seule unité. Il ajoute que son père ne leur a jamais rien imposé; au contraire, la décision finale revient toujours au dirigeant responsable de l'activité.

D'après les deux dirigeants interviewés, le choix des activités est arbitraire. La diversification permet de contrôler la filiale de production et d'améliorer leur position sur le marché, alors que la création de petites unités permet de mieux maîtriser la croissance. Trois des activités de ces unités sont intégrées, une activité étant liée au domaine d'origine et une autre totalement diversifiée. Cette dernière peut être considérée comme un moyen de loisir pour le père fondateur. L'indépendance des entreprises facilite le désengagement en cas d'échec sans toucher à l'activité d'origine. Cette stratégie réduit le risque de la diversification, augmente le pouvoir de marché et permet d'exploiter des synergies commerciales et relationnelles.

Cas 5. Cet hypogroupe, opérant dans divers secteurs d'activité, a été créé à l'origine par le père fondateur. Ensuite, prenant en charge son neveu, ce fondateur a développé une nouvelle activité dont les compétences nécessaires correspondaient au profil de ce membre de la famille qui la dirige désormais. 
Afin que ces successeurs soient en mesure de prendre la relève et d'intégrer le management de l'entreprise familiale, ce fondateur a reproduit le même modèle de développement. Nous avons pu obtenir un rendez-vous avec ce fondateur et avec son fils unique le successeur (4). Actuellement, chaque successeur dirige une unité de l'hypogroupe présenté par le tableau suivant.

TABLEAU 6

Présentation des unités de l'hypogroupe 5

\begin{tabular}{lccccc}
\hline & $\begin{array}{c}\text { Date de } \\
\text { création }\end{array}$ & Effectif & Activité & Produit & Dirigeant \\
\hline $\begin{array}{l}\text { Unité 1 } \\
\text { (entreprise } \\
\text { d'origine) }\end{array}$ & 1979 & 21 & Services & $\begin{array}{c}\text { Transit } \\
\text { maritime }\end{array}$ & Fondateur 1 \\
\hline Unité 2 & 1983 & 12 & Agroalimentaire & $\begin{array}{c}\text { Entreposage } \\
\text { de l'huile } \\
\text { d'olive }\end{array}$ & $\begin{array}{c}\text { Dirigeant } \\
\text { membre de } \\
\text { la famille } \\
\text { (neveu) }\end{array}$ \\
\hline Unité 3 & 1989 & 35 & Industrie textile & $\begin{array}{c}\text { Traitement } \\
\text { des articles } \\
\text { confectionnés }\end{array}$ & $\begin{array}{c}\text { Successeure 1 } \\
\text { (fille aîné) }\end{array}$ \\
\hline Unité 4 & 1991 & 10 & Services & $\begin{array}{c}\text { Location } \\
\text { de bascules }\end{array}$ & $\begin{array}{c}\text { Successeure 2 } \\
\text { (2e fille) }\end{array}$ \\
\hline Unité 5 & 1995 & 50 & Services & $\begin{array}{c}\text { Services } \\
\text { pétroliers }\end{array}$ & $\begin{array}{c}\text { Successeure } \\
\text { 3 (3 fille) + } \\
\text { professionnel }\end{array}$ \\
\hline Unité 6 & 1999 & 15 & Services & Manutention & $\begin{array}{c}\text { Successeur 4 } \\
\text { (fils unique) }\end{array}$ \\
\hline
\end{tabular}

Cette famille est de type égalitaire. L'attention portée à l'égalité avait incité le fondateur à développer l'hypogroupe afin d'assurer à ses enfants les mêmes conditions de travail et les mêmes avantages. Quant à l'autorité, le fondateur est présumé autoritaire selon le successeur (4); il n'autorise aucune négociation. Ainsi, lorsque les deux premiers successeurs (filles) ont bénéficié de leur indépendance à travers la création de nouvelles unités, les autres se sont sentis lésés bien que le fondateur leur eût accordé la responsabilité de l'entreprise d'origine. Ce sentiment était dû au fait que l'objectif d'indépendance des successeurs ne pouvait pas être atteint compte tenu de l'autorité du prédécesseur.

Cependant, l'égalité pour le fondateur revient à accorder à chaque membre les parts de capital qui traduisent le poids de ses responsabilités. Plus le successeur a de tâches à accomplir, plus sa part de capital est importante. Il considère que cette règle de partage motive les membres de la famille et essentiellement les successeurs. En fait, il offre à chacun les mêmes opportunités de développement. À ses yeux, cette stratégie est égalitaire. Cette même 
stratégie est, selon le successeur (4), une source de conflits. Ainsi, toujours dans un objectif d'égalité et pour corriger l'erreur de départ, le fondateur développe de nouveaux domaines d'activité indépendants.

Les successeurs sont indépendants mais pas de leur père: il est toujours le propriétaire majoritaire sans laisser cours au dialogue et à la négociation. Son autorité fait qu'il décide lui-même du déclenchement du processus de transmission et de ses acteurs. Cela n'empêche que son équité adoucit un peu son autorité. Son désir d'assurer l'égalité l'a amené à créer des champs d'autonomie à ses successeurs.

Les activités ne sont pas vraiment choisies. Le successeur (4) estime qu'il n'avait pas d'autres alternatives pour faire le tri. Le développement était plutôt émergent sans planification préalable et ce n'est que récemment que les dirigeants commencent à structurer et à planifier les activités de l'hypogroupe. En revanche, pour le neveu, la diversification consiste à être présent sur plus d'un secteur d'activité. Cette stratégie était à la fois imposée par le profil des successeurs et recherchée pour atténuer les risques.

Essentiellement, la diversification totale est justifiée par les qualifications des successeurs qui dirigent les unités et par le fait qu'ils ne peuvent pas assumer la direction de l'entreprise d'origine. De fait, le fondateur leur a créé des unités qui correspondent davantage à leurs profils.

\subsection{L'analyse du développement des hypogroupes familiaux : une analyse inter-cas}

L'analyse de contenu de ces cas nous permet d'avancer que le développement en hypogroupe est lié à la structure de la famille, notamment à l'égalité. L'égalité permet d'éviter les conflits potentiels entre successeurs et, par conséquent, favorise l'atteinte des objectifs de pérennité et de maintien du contrôle familial de la propriété et du management. Afin de discuter ces résultats, nous allons examiner l'impact de la structure de la famille sur le développement en hypogroupe. Ensuite, nous montrerons que la formation de l'hypogroupe est inspiré par une stratégie de diversification.

\subsubsection{La structure de la famille}

L'égalité se manifeste à travers l'égalité dans l'héritage (même pourcentage), l'égalité des sexes (femme et homme) et l'égalité des chances (pour accorder des responsabilités de gestion aux successeurs ou pour les aider à atteindre leurs objectifs personnels). Le tableau suivant permet de classer les cas étudiés selon ces critères. 
TABLEAU 7

\section{Classification des cas selon les critères d'égalité}

\begin{tabular}{|c|c|c|c|c|c|}
\hline Critères & Cas 1 & Cas 2 & Cas 3 & Cas 4 & Cas 5 \\
\hline Égalité dans & $\begin{array}{l}\text { Plusieurs } \\
\text { successeurs. }\end{array}$ & $\begin{array}{l}\text { Plusieurs } \\
\text { successeurs. }\end{array}$ & $\begin{array}{l}\text { Plusieurs } \\
\text { successeurs. }\end{array}$ & $\begin{array}{l}\text { Plusieurs } \\
\text { successeurs. }\end{array}$ & $\begin{array}{l}\text { Plusieurs } \\
\text { successeurs. }\end{array}$ \\
\hline l'héritage & $\begin{array}{l}\text { Mêmes parts } \\
\text { de capital. }\end{array}$ & $\begin{array}{l}\text { Mêmes parts } \\
\text { de capital. }\end{array}$ & $\begin{array}{l}\text { Mêmes parts } \\
\text { de capital. }\end{array}$ & $\begin{array}{l}\text { Mêmes parts } \\
\text { de capital. }\end{array}$ & $\begin{array}{l}\text { Mêmes parts } \\
\text { de capital. }\end{array}$ \\
\hline $\begin{array}{l}\text { Égalité } \\
\text { des sexes }\end{array}$ & $\begin{array}{l}\text { Femme } \\
\text { et homme }\end{array}$ & - & - & $\begin{array}{l}\text { Femme } \\
\text { et homme }\end{array}$ & $\begin{array}{l}\text { Femme } \\
\text { et homme }\end{array}$ \\
\hline $\begin{array}{l}\text { Égalité } \\
\text { des chances }\end{array}$ & Oui & Oui & Oui & Oui & Oui \\
\hline
\end{tabular}

L'héritage est partagé à égalité entre tous les successeurs (pas d'héritier unique). Qu'ils soient femme ou homme, les successeurs ont droit au capital de l'entreprise familiale et sont impliqués au management de celle-ci. Pour les cas (2) et (3), la famille est composée de garçons, ce qui ne permet pas de se prononcer sur l'égalité des sexes. Cependant, tous les fondateurs de tous les cas offrent à tous les successeurs les mêmes chances de réalisation personnelle et de développement des activités qui correspondent à leurs aspirations.

Ces cas sont de type égalitaire mais se différencient au regard du critère liberté/autorité comme le montre le tableau suivant.

TABLEAU 8

Classification des cas selon le critère liberté/autorité

\begin{tabular}{lccccc}
\hline Critères & Cas 1 & Cas 2 & Cas 3 & Cas 4 & Cas 5 \\
\hline Liberté des enfants & & & $\mathrm{X}$ & $\mathrm{X}$ & \\
\hline Autorité du père & $\mathrm{X}$ & $\mathrm{X}$ & & & $\mathrm{X}$ \\
\hline
\end{tabular}

En fait, la liberté de la famille (3) est sans effet sur le développement en hypogroupe. C'est plutôt l'égalité, tout comme pour le cas (4), qui avait poussé ses dirigeants à créer de nouvelles unités pour que chaque successeur bénéficie des mêmes avantages et de la même liberté d'action. Par contre, l'autorité des fondateurs des cas (1),(2) et (5) était à l'origine de la recherche d'autonomie et de liberté d'action de la part des successeurs. L'égalité avait atténué les tensions entre les différentes générations puisque les fondateurs autoritaires étaient conscients du fait que chaque successeur avait le droit à la même indépendance que les autres, ce qui n'était pas simple à assurer au sein d'une seule unité. Ces fondateurs avaient donc décidé de créer de nouvelles unités.

Ainsi, l'étude de l'égalité en tant que caractéristique de la structure de la famille qui affecte le partage du patrimoine et donc la transmission 
nous a enseigné que ce n'est pas l'atteinte du consensus autour du résultat du partage des biens qui est importante mais bien la manière avec laquelle les membres de la famille s'y sont pris pour l'obtenir. Ce qui est important à retenir en fin de cette étude, c'est que les dirigeants-propriétaires ont choisi le développement en hypogroupe dans le but d'assurer une plus grande égalité.

Ce processus de développement menant à l'égalité (du moins perçue) reconnaît que les prédécesseurs ont, plus que les autres, une plus grande responsabilité sur la pérennité de l'entreprise familiale. Un processus de prise de décision mené de manière juste et équitable ne nie pas l'autorité mais s'intéresse plutôt à la façon dont l'autorité est exercée (Taylor et Norris, 2000).

Un processus de transmission juste contribue même à augmenter la satisfaction des membres de la famille et l'engagement des générations futures comme cela a été souligné dans les travaux de Van der Hayden, Blondel et Carlock (2005). Ces auteurs affirment que l'égalité engendre moins de conflits et que des relations familiales plus proches font qu'il y a peu ou pas de conflits ou qu'il y a plus de possibilité de résoudre les conflits avec satisfaction. Par conséquent, la structure de la famille communautaire affecte le développement de l'entreprise familiale en hypogroupe. La répartition des responsabilités de gestion est égalitaire entre les enfants d'une même génération. La première proposition est alors non rejetée puisque, d'après les résultats, l'égalité est l'un des déterminants de la création de nouvelles unités familiales.

\subsubsection{La stratégie de diversification}

Le développement des cas étudiés était conduit par une stratégie de diversification. Pour les cas (1), (4), et (5), la diversification est le résultat de la gestion des relations familiales entre les membres. En fait, la prévention des problèmes et la préparation du processus de transmission poussent les fondateurs à multiplier les unités bien que l'objectif de contrôle de propriété et de management leur interdise de trop s'écarter de leur domaine de compétence d'origine. Les activités répondant aux aspirations des membres qui avaient des compétences et des profils très variés, le résultat est alors une diversification totale dans des activités choisies par les membres qui les dirigent pour répondre à leurs besoins.

Quant aux cas (2) et (3), ils ont adopté plusieurs types de diversification pour répondre à la fois aux besoins de la famille et à ceux de l'activité économique. La première diversification de ces hypogroupes visait la préservation du patrimoine familial qui était menacé par les contraintes de l'environnement économique. Les successeurs sont venus en temps opportun pour remédier à ces situations qui menaçaient la pérennité de leurs entreprises familiales. 
Ainsi, le choix de la stratégie de diversification vise, selon les cas, deux types d'objectifs :

- un objectif familial lié à la pérennité de l'entreprise;

- un objectif économique lié à l'exploitation de synergies et à la construction d'avantage concurrentiel.

En fait, pour ce qui est du premier objectif, la diversification est plutôt non liée au domaine d'origine. Elle est souvent choisie comme voie de développement lorsque les successeurs résistent manifestement à la reprise de l'unité d'origine. Dans certains cas étudiés, le successeur se trouve obligé d'assurer la relève contre sa volonté, ce qui conduit généralement à un raccourcissement des phases de préparation et de planification de la succession ou à leur inexistence (Barbot et Richomme-Huet, 2003). Le successeur doit ainsi montrer son engagement, son attachement et son implication envers l'entreprise (Fattoum et Fayolle, 2005). Dans ce cas, les dirigeants diversifient les activités afin d'attirer les successeurs et leur permettre de réaliser ce qu'on attend d'eux. C'est pourquoi la deuxième proposition stipulant que le développement par la voie de la diversification conduit au choix de l'organisation stratégique en hypogroupe est non rejetée.

Par conséquent, la flexibilité de la forme d'hypogroupe et le fait qu'elle permet de s'adapter à différents besoins organisationnels, notamment la centralisation et l'autonomie, sont d'importantes raisons qui expliquent le choix du développement de l'hypogroupe familial (Iacobucci, 2002).

Les relations que nous avons établies entre la structure de la famille et le développement de l'entreprise familiale par création de nouvelles unités conformément aux propositions formulées ci-dessus peuvent être schématisées comme suit:

FIGURE 2

L'impact de la structure de la famille sur le développement en hypogroupe

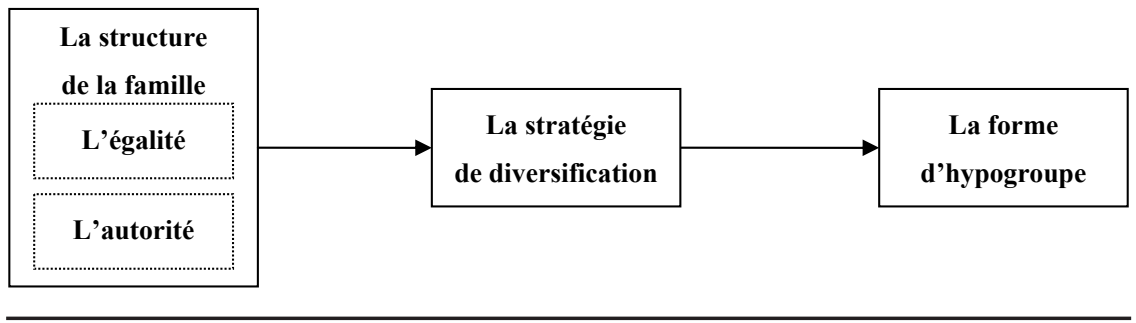


La famille est ainsi un système social qui prend soin de ses membres et développe le sens de loyauté envers ces derniers. Les liens sociaux qui animent le système familial proviennent du rôle que joue ce dernier dans la satisfaction des besoins économiques et sociaux de ses membres. Cette allocation des ressources au sein de la famille se structure autour de normes partagées d'égalité.

\section{Conclusion}

Le choix de la structure d'hypogroupe reflète majoritairement une recherche de développement minimisant le risque de déstabilisation de l'unité familiale d'origine, inhérent à la délégation du contrôle managérial. Ainsi, les dirigeants-propriétaires créent de nouvelles unités dont les finalités correspondent aux aspirations des membres concernés. Un tel comportement s'applique d'une façon uniforme sans privilégier certains par rapport à d'autres grâce à une structure familiale égalitaire. Cette caractéristique fait que la répartition des responsabilités de gestion est égalitaire entre les enfants d'une même génération. Cette égalité est susceptible d'éviter les conflits entre les successeurs en leur accordant les mêmes avantages, ce qui nécessite la création de multiples entreprises indépendantes. Ainsi, les relations au sein de la famille sont gouvernées par des critères (valeurs partagées, anticipations, etc.) qui ressortent plus de l'émotion que de la rationalité (Gomez-Mejia, Larraza-Kintana et Makri,2003). Les gains économiques ou les profits ne sont pas de ce fait les seuls moteurs de l'activité au sein de l'entreprise familiale (Coeurderoy et Lwango, 2008).

Cependant, d'une manière générale, les résultats d'une recherche sont d'autant plus exploitables que leurs limites sont admises. Pour ce qui est de cette recherche, nous lui reconnaissons deux principales limites. La première concerne les possibilités d'amélioration que l'on pourrait apporter à la conceptualisation du développement en hypogroupe et au choix des variables retenues pour le modèle. Plus précisément, ce choix que nous avons fait en faveur de certaines variables plutôt que d'autres gagnerait à être consolidé par une étude plus approfondie. La deuxième limite renvoie à la taille de notre échantillon. En fait, ce sont des contraintes d'accès au terrain ainsi que des limites de temps qui nous ont empêchés d'étudier un nombre plus important de cas.

Les limites précédemment évoquées permettent d'envisager différentes pistes de recherche. La voie de recherche la plus évidente sera la réplication de l'étude sur un échantillon d'hypogroupes dans un contexte différent du contexte tunisien. Ce type de démarche permettrait de confirmer ou d'infirmer les résultats auxquels nous sommes arrivés. Il serait aussi intéressant 
de réaliser une étude quantitative dans le but de construire et de vérifier certaines hypothèses. Maintenant que nous disposons d'un modèle, il nous semblerait intéressant de poursuivre notre étude par une recherche hypothéticodéductive. Sur le plan pratique, l'intérêt réside dans l'accompagnement des cas étudiés. L'étude de la dynamique de ces hypogroupes fait partie de nos préoccupations de recherches futures.

\section{Bibliographie}

Allouche, J., B. Amann et P. Garaudel (2006), «Performances et caractéristiques financières des entreprises familiales et non familiales: le rôle modérateur de la cotation en bourse et du degré de contrôle actionnarial», Les cahiers de recherche du GREGOR $n^{\circ}$ 02, Université de Paris I, Panthéon Sorbonne.

BANQUE MONDIALE (2000), «République de Tunisie: mise à jour de l'évaluation du secteur privé», Rapport n ${ }^{\circ}$ 200173-tun, décembre.

BArbot, M.C. et K. Richomme-Huet (2003), «Le contexte successoral des PME familiales implique-t-il un processus entrepreneurial?», Colloque AIREPME «L'entrepreneuriat en action: contextes et pratiques », 23 et 24 octobre, Agadir.

BARRÉDY, C. (2002), «Structures juridiques, gouvernance et développement de l'entreprise», dans G. Hirigoyen et J. Caby (dir.), La gestion des entreprises familiales, Paris, Economica.

BASLY, S. (2005), L'internationalisation de la PME familiale: une analyse fondée sur l'apprentissage organisationnel et le développement de la connaissance, Thèse de doctorat en sciences de gestion, Université Montesquieu Bordeaux IV.

Blanchet, A. et A. Gotman (1992), L'enquête et ses méthodes: l'entretien, Paris, Nathan Université.

Boissin, J.P. (2000), «Système de valeurs du management familiale en PME», $\mathrm{V}^{\mathrm{e}}$ Congrès international francophone en entrepreneuriat et PME, $25 \mathrm{au}$ 27 octobre, Lille, p. 1-16.

Bourdieu, P. (1994), Raisons sociales: Essais, Paris, Éditions du Seuil.

BRUNNER, C. (1975), «Les contraintes de la fusion dans le secteur des PME», Analyse financière, $3^{\text {e }}$ trimestre, p. 103-106.

CADIEuX, L. et J. LoRRAIN (2002), «Le processus de la succession dans les entreprises familiales: une problématique comportant des défis estimables pour les chercheurs », $\mathrm{VI}^{\mathrm{e}}$ Congrès international francophone sur la PME, octobre, HEC Montréal.

CHElly, A. (2006), Les processus de génération d'opportunités entrepreneuriales: une recherche exploratoire auprès d'entrepreneurs tunisiens, Thèse de doctorat, Université du Sud Toulon Var.

Chrisman, J.J., J.H. CHUA et L.P. STEIER (2003), «An introduction to theories of family business », Journal of Business Venturing, vol. 18, n 4, p. 441-448. 
Chua, J.H, J.J. Chrisman et P. Sharma (1999), «Defining the family business by behaviour», Entrepreneurship. Theory and Practice, vol. 23, n 4, p. 19-36.

Couerderoy, R. et A. LWANGo (2008), «Capital social, coût de mesure et efficacité organisationnelles: y a-t-il un avantage spécifique à l'entreprise familiale?», Louvain School of Management, Document de travail 04.

Crémadez, M. (2004), Organisations et stratégie, Paris, Dunod.

DAILY, C. et M. Dollinger (1992), «An empirical examination of ownership structure in family and professionally managed firm », Family Business Review, vol. $5, \mathrm{n}^{\circ}$ 2, p. 117-136.

Debray, C. (1997), Structure et stratégies de l'hypogroupe, Thèse de doctorat, Université de Montpellier, Faculté de gestion et de droit de l'entreprise.

DEBRAY, C. et C. LEYRONAS (1996), «Réseau et hypogroupe: émergence de stratégies organisationnelles en petite entreprise », XIII ${ }^{\text {es }}$ Journées nationales des IAE, 16 et 17 avril, Toulouse.

Defreyman, J., K. Richomme-Huet et R. PATUREL (2006), «Les obstacles au transfert du réseau social dans la transmission de l'entreprise artisanale familiale », $\mathrm{XV}$ Conférence internationale de management stratégique, Annecy/Genève, 13-16 juin.

DESLAURIERS, J.P. et M. KÉRESIT (1997), «Le devis de recherche qualitative », dans J. Poupart et al. (dir.), La recherche qualitative: enjeux épistémologiques et méthodologiques, Boucherville, Gaëtan Morin Éditeur, p. 85-112.

DunN, B. (1996), «Family enterprise in the U.K: a special sector», Family Business Review, vol. 9, n 2, p. 139-155.

DYER, W. G. (2003), «The family: the missing variable in organizational research», Entrepreneurship. Theory and Practice, été, p. 401-416.

EISENHARDT, K. (1989), «Building theories from case study research », Academy of Management Review, vol. 14, n 4, p. 532-550.

FATTOUM, S. et A. FAYOLLE (2005), «L'impact de la relation prédécesseur/successeur sur le déroulement du processus de succession dans les entreprises familiales», IV ${ }^{\mathrm{e}}$ Congrès de l'Académie de l'entrepreneuriat, Paris, Sénat, 24-25 novembre.

GAUlTIER, A. (1990), Les holdings familiales : pérennité et succession, Paris, Éditions de l'Organisation.

GOMEZ-MEJIA, L.R., M. LARRAZA-KinTANA et M. MAKRI (2003), «The determinants of executive compensation in family-controlled public corporations », Academy of Management Journal, vol. 46, n 2, p. 226-237.

GranovetTER, M. (2005), «Business groups and social organization», dans N.J. Smelser et R. Swedberg (dir.), The Handbook of Economic Sociology, Princeton, N.J., Princeton University Press.

HADDADJ, S. et A. ANDRIA (2001), «Mesurer les phénomènes agissant sur la transmission des PME familiale », Revue française de gestion, $\mathrm{n}^{\circ} 132$, janvier-février, p. 11-22. 
Huppert, R. (1981), «Stratégies de développement des PMI françaises », Revue d'économie industrielle, $\mathrm{n}^{\circ}$ 17, $3^{\mathrm{e}}$ trimestre, p. 26-42.

IACOBUCCI, D. (2002), «Explaining business groups started by habitual entrepreneurs in the Italian manufacturing sector », Entrepreneurship and Regional Development, vol. 14, n 1 , p. 31-48.

IACOBUCCI, D. et P. ROSA (2005). « Growth, diversification, and business group formation in entrepreneurial firms », Small Business Economics, vol. 25, n 1, p. 65-82.

Julien, P.-A. et M. MARCHESNAY (1987), La petite entreprise, Paris, Vuibert.

KALIKA, M. (1988), Structures d'entreprises: réalités, déterminants, performances, Paris, Gestion, Economica.

Kellermanns, F.W. et K.A. EddLESTON (2006), «Corporate entrepreneurship in family firms: a family perspective», Entrepreneurship. Theory and Practice, vol. $30, n^{\circ} 6$, p. 809-830.

LEYRONAS, C. (2004), «Processus de transformation d'un réseau par hypogroupement», VII ${ }^{\mathrm{e}}$ Congrès international francophone en entrepreneuriat et PME, 27 au 29 octobre, Montpellier.

MARCHESNAY, M. (1993), Management stratégique, Paris, Eyrolles.

MignON, S. (2000), «La pérennité des entreprises familiales: un modèle alternatif à la création de valeur pour l'actionnaire », Finance, Contrôle et Stratégie, vol. 3, $\mathrm{n}^{\circ}$ 1, p. 169-196.

Miles, M.B. et A.M. Huberman (2003), Analyse des données qualitatives : recueil de nouvelles méthodes, $2^{\mathrm{e}}$ édition, De Boeck, Bruxelles.

MintzBerg, H. (1982), Structure et dynamique des organisations, Paris, Éditions d'Organisation.

MzID, I. et L. MEZGHANI (2008), «La transmission de la direction et le développement de l'entreprise familiale en hypogroupe », XVII ${ }^{\mathrm{e}}$ Conférence de l'Association internationale de management stratégique (AIMS), du 28 au 31 mai, Nice.

NABLI, R. (2009), «La vulnérabilité des TPE et des PME dans un environnement

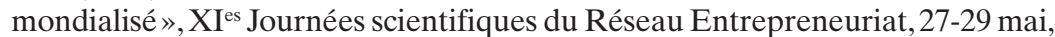
Trois-Rivières, Québec (Canada).

RisPAL, H. (2002), La méthode des cas: application à la recherche en gestion, De Boeck Université, Belgique.

RosA, P. (1998), «Entrepreneurial process of business cluster formation and growth by habitual entrepreneurs », Entrepreneurship. Theory and Practice, vol. 22, $\mathrm{n}^{\circ}$ 4, p. 43-62.

RosA, P. et M. SCOTT (1999), «Entrepreneurial diversification, business cluster formation and growth », Environment and Planning, vol. 17, n 5, p. 527-548.

SHARMA, P. (1997), Determinants of the Satisfaction of the Primary Stakeholders with the Succession Process in Family Firms, Thèse de doctorat, University of Calgary. 
SHARMA, P. et S. MANIKUTTY (2003), «Shedding of unproductive resources in family firms: role of family structure and community culture», The School of Business and Economics Publishes Working Papers.

TAYLOR, J.E. et J.E. NORRIS (2000), « Sibling relationships, fairness, and conflict over transfer of the farm », Family Relations, vol. 49, n 3, p. 277-283.

THIÉTART, R.A. et al. (2004), Méthodes de recherche en management, $2^{\mathrm{e}}$ édition, Paris, Dunod.

TODD, E. (1983), La troisième planète: structures familiales et systèmes idéologiques, Paris, Seuil.

VAN DER HEYDEN, L., C. BLONDEL et R.S. CARLOCK (2005), «Fair process: striving for justice in family business », Family Business Review, vol. 18, n 1, p. 1-18

VAS, A. (2005), «Le processus de changement organisationnel à l'épreuve des faits: une approche multiparadigmatique», Management international, hiver, vol. 9, $\mathrm{n}^{\circ} 2$, p. 21-36.

WARD, J.L. (2005), «La gouvernance des entreprises familiales», E Journal USA: perspectives économiques, février, p. 42-45.

Yin, R. (1994), Case Study Research: Design and Methods, Beverly Hills, Sage Publications.

ZGHAL, R. (1994), La culture de la dignité et le flou organisationnel, CPU, Tunis. 